Proceedings of the ISCIE International Symposium on Stochastic Systems Theory and Its Applications Osaka, Nov. 10-12, 1993

\title{
Identification of Multi-Input, Multi-Output Continuous Time-Delay Systems Combining the Instrumental Variable Method with the Genetic Algorithm
}

\author{
Tomohiro Hachino, Zi-Jiang Yang and Teruo Tsuji \\ Department of Electrical Engineering \\ Kyushu Institute of Technology
}

\begin{abstract}
This paper proposes a new method of identification of continuous time-delay systems from sampled input-output data. Identification of multiinput, multi-output systems where the time-delays in the individual input channels may differ from each other, is discussed. With the aid of a digital pre-filter, an approximated discrete-time estimation model is first derived at, in which the system parameters remain in their original form and the time-delays need not be an integral multiple of the sampling period. Then an identification method combining the instrumental variable (IV) method with the genetic algorithm (GA) is proposed, i.e the time-delays are selected by the GA, and the system parameters are estimated by the IV method. Simulation results show that our method yields excellent estimates even in the presence of high measurement noises.
\end{abstract}

\section{Introduction}

Since many practical systems have inherent delays, the problem of identifying such a system is of great importance for analysis, synthesis and prediction. However, the identification of time-delay systems is greatly complicated, because the estimation model becomes nonlinear in the parameters with respect to the time-delays.

Numerous identification methods of time-delay systems based on the discrete-time model have been proposed $^{1) ~ 3)}$. In the case of the discrete-time model, the sampling period is required to be very small as the time-delays are an integral multiple of the sampling period, whereas if the sampling period is too small, the identification becomes very difficult. Moreover, the parameters in the discrete-time model usually do not correspond to the physical parameters. Therefore, the importance of the identification based on the continuous-time model has been recognized in recent years ${ }^{4)}$. We also consider the identification of time-delay systems based on the continuous-time model.

There are two typical approaches based on the continuous-time model. One approach is based on the approximation of the time-delays in the frequency domain by a rational transfer function or the padé approximation ${ }^{5) ~ 7)}$. This approach requires an estimation of more parameters because the order of the approximated system model is increased, and it is not easy to separate the parameters concerned with the time-delays from those with the dynamics of the system. Moreover an unacceptable approximation error occurs when the system has large time-delays.

The other approach is based on the nonlinear estimation method like the nonlinear least squares method etc. $^{8) ~ 12)}$. In this approach, unfortunately the estimates of the time-delays and the system parameters often converge to local optima if we do not choose the suitable initial values for the parameters and forgetting factors. Moreover, this method is infeasible when the measurements are corrupted by high level noises.

In the above mentioned works, the identification of time-delay systems which have multi time-delays are seldom considered. Since the identification of such a system becomes more difficult, to the best of the 
authors' knowledge, few works which deal with the identification of multi-input, multi-output time-delay systems have been reported.

The GA is a probabilistic search procedure based on the mechanics of natural selection and natural genetics ${ }^{13)}$. Recently the GA has received considerable attention in various fields ${ }^{14), 15)}$, because it has a high potential for global optimization.

In this paper, we propose a method of identification of continuous time-delay systems from sampled input-output data combining the IV method with the GA. At first, with the aid of a digital pre-filter, we get an approximated discrete-time estimation model, in which the system parameters remain in their original form, and the time-delays need not be an integral multiple of the sampling period. Then, candidates of the unknown time-delays in this model are coded into binary bit strings, and the system parameters are estimated based on the IV method using each of the candidates of the decoded time-delays which are selected by the GA. Furthermore, reproduction, crossover and mutation are repeated so that the average fitness value of the population increases. In this case, the fitness values are represented by the function of the mean squares errors which are calculated by using the estimates of the parameters. Thus the estimates of the time-delays and the system parameters can be obtained. It is shown by simulations that the method combining the IV method with the GA gives consistent estimates even in the presence of high measurement noises.

\section{Statement of the problem}

Consider the following multi-input, multi-output continuous time-delay system:

$$
\begin{aligned}
& {\left[\begin{array}{c}
x_{1}(t) \\
\vdots \\
\vdots \\
x_{q}(t)
\end{array}\right]=\left[\begin{array}{cccc}
\frac{N_{11}(p)}{D_{1}(p)} & \frac{N_{12}(p)}{D_{1}(p)} & \cdots & \frac{N_{1 r}(p)}{D_{1}(p)} \\
\vdots & \vdots & \ddots & \vdots \\
\frac{N_{q 1}(p)}{D_{q}(p)} & \frac{N_{q 2}(p)}{D_{q}(p)} & \cdots & \frac{N_{q r}(p)}{D_{q}(p)}
\end{array}\right]\left[\begin{array}{c}
u_{1}\left(t-\tau_{1}\right) \\
\vdots \\
\vdots \\
u_{r}\left(t-\tau_{r}\right)
\end{array}\right]} \\
& D_{i}(p)=\sum_{j=0}^{n} a_{i j} p^{n-j} \quad\left(a_{i 0}=1 ; i=1,2, \cdots, q\right) \\
& N_{i h}(p)=\sum_{j=1}^{m} b_{i h j} p^{m-j} \quad(i=1,2, \cdots, q ; h=1,2, \cdots, r)
\end{aligned}
$$

Eq.(1) can be written as a set of multi-input, single-output systems:

$$
\sum_{j=0}^{n} a_{i j} p^{n-j} x_{i}(t)=\sum_{h=1}^{r} \sum_{j=1}^{m} b_{i h j} p^{m-j} u_{h}\left(t-\tau_{h}\right) \quad\left(a_{i 0}=1\right) \quad(i=1,2, \cdots, q)
$$

where $p$ is a differential operator, $u_{h}(t), x_{i}(t)$ are the $h$ th input and the $i$ th real output, and $\tau_{h}$ is the time-delay in the $h$ th input. $n$ and $m$ are assumed to be known $(n \geq m)$.

It is assumed that a zero-order hold is utilized such that

$$
u_{h}(t)=\bar{u}_{h}(k) \quad(k-1) T \leq t<k T \quad(h=1,2, \cdots, r)
$$

where $T$ is the sampling period.

Practically the measurements of the output variables are corrupted by stochastic measurement noises.

$$
y_{i}(k)=x_{i}(k)+v_{i}(k) \quad(i=1,2, \cdots, q)
$$

Our goal is to identify the time-delays and the system parameters from sampled data of the inputs and noisy outputs. 


\section{Approximated discrete-time estimation model}

An approximated discrete-time estimation model is obtained with the aid of a digital pre-filter in order to avoid the direct signal derivatives. In this paper we introduce a low-pass pre-filter $Q_{i}(p)$ for each subsystem as

$$
Q_{i}(p)=\frac{1}{\left(\alpha_{i} p+1\right)^{n}} \quad(i=1,2, \cdots, q)
$$

where $\alpha_{i}$ is the time constant which determines the pass-band of $Q_{i}(p)$.

Multipling both sides of Eq.(2) by $Q_{i}(p)$ and using the bilinear transformation based on the block-pulse functions ${ }^{4), 16), 17)}$, we can obtain the following approximated discrete-time estimation model of the original

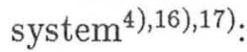

$$
\xi_{0 \bar{y}_{i}}(k)+\sum_{j=1}^{n} a_{i j} \xi_{j \bar{y}_{i}}(k)=\sum_{h=1}^{r} \sum_{j=1}^{m} b_{i h j} \xi_{(n-m+j) \bar{u}_{h}^{i}}\left(k-\tilde{\tau}_{h}\right)+r_{i}(k) \quad(i=1,2, \cdots, q)
$$

where for $i=1,2, \cdots, q$

$$
\begin{aligned}
\xi_{j \bar{y}_{i}}(k) & =Q_{i 0}\left(z^{-1}\right)\left(\frac{T}{2}\right)^{j}\left(1+z^{-1}\right)^{j}\left(1-z^{-1}\right)^{n-j} \bar{y}_{i}(k) \\
\xi_{(n-m+j) \bar{u}_{h}^{i}}\left(k-\tilde{\tau}_{h}\right) & =Q_{i 0}\left(z^{-1}\right)\left(\frac{T}{2}\right)^{n-m+j}\left(1+z^{-1}\right)^{n-m+j}\left(1-z^{-1}\right)^{m-j} \bar{u}_{h}\left(k-l_{h}\right) \\
r_{i}(k) & =\sum_{j=0}^{n} a_{i j} Q_{i 0}\left(z^{-1}\right)\left(\frac{T}{2}\right)^{j}\left(1+z^{-1}\right)^{j}\left(1-z^{-1}\right)^{n-j} v_{i}(k) \\
Q_{i 0}\left(z^{-1}\right) & =\frac{1}{\left[\alpha_{i}\left(1-z^{-1}\right)+\frac{T}{2}\left(1+z^{-1}\right)\right]^{n}}
\end{aligned}
$$

Here $\bar{y}_{i}(k)=\left(1+z^{-1}\right) y_{i}(k) / 2$ is the block-pulse approximation of $y_{i}(t)^{4), 16), 17)}$ and $\tilde{\tau}_{h}$ is given by

$$
\tilde{\tau}_{h}=\tau_{h} / T=l_{h}+\Delta_{h} / T \quad\left(0 \leq \Delta_{h}<T \quad ; \quad l_{h} \text { : nonnegative integer }\right)
$$

It should be noticed that generally it is desirable that the time-delays $\tau_{h}(h=1,2, \cdots, r)$ are an integral multiple of the sampling period in the identification based on the discrete-time model, whereas our approximated discrete-time estimation model does not require this restriction. Because in the case of $\Delta_{h} \neq 0$, we can get $\xi_{(n-m+j) \bar{u}_{h}^{i}}\left(k-\tilde{\tau}_{h}\right)$ by the linear interpolation of $\xi_{(n-m+j) \bar{u}_{h}^{i}}\left(k-l_{h}\right)$ and $\xi_{(n-m+j) \bar{u}_{h}^{i}}\left(k-l_{h}-1\right)$.

Eq.(6) can be written in vector form :

$$
\xi_{0 \bar{y}_{i}}(k)=\mathrm{z}_{i}^{T}\left(k, \tilde{\tau}_{1}, \cdots, \tilde{\tau}_{r}\right) \boldsymbol{\theta}_{i}+r_{i}(k) \quad(i=1,2, \cdots, q)
$$

where

$$
\begin{aligned}
\mathrm{z}_{i}^{T}\left(k, \tilde{\tau}_{1}, \cdots, \tilde{\tau}_{r}\right) & =\left[-\mathbf{z}_{i \bar{y}}^{T}(k), \mathbf{z}_{i \bar{u}_{1}}^{T}\left(k-\tilde{\tau}_{1}\right), \cdots, \mathrm{z}_{i \bar{u}_{r}}^{T}\left(k-\tilde{\tau}_{r}\right)\right] \\
\mathbf{z}_{i \bar{y}}^{T}(k) & =\left[\xi_{1 \bar{y}_{i}}(k), \cdots, \xi_{n \bar{y}_{i}}(k)\right] \\
\mathbf{z}_{i \bar{u}_{h}}^{T}\left(k-\tilde{\tau}_{h}\right) & =\left[\xi_{(n-m+1) \bar{u}_{h}^{i}}\left(k-\tilde{\tau}_{h}\right), \cdots, \xi_{n \bar{u}_{h}^{i}}\left(k-\tilde{\tau}_{h}\right)\right] \quad(h=1,2, \cdots, r) \\
\boldsymbol{\theta}_{i}^{T} & =\left[\boldsymbol{a}_{i}^{T}, \boldsymbol{b}_{i 1}^{T}, \cdots, \boldsymbol{b}_{i r}^{T}\right] \\
\boldsymbol{a}_{i}^{T} & =\left[a_{i 1}, a_{i 2}, \cdots, a_{i n}\right] \\
\boldsymbol{b}_{i h}^{T} & =\left[b_{i h 1}, b_{i h 2}, \cdots, b_{i h m}\right] \quad(h=1,2, \cdots, r)
\end{aligned}
$$




\section{Identification of the system parameters}

Before presenting the identification method of the parameters including the time-delays, in this section we describe only the identification of the system parameters in the case of the known time-delays.

From Eq.(9) the system parameters can be estimated by the following least squares (LS) method:

$$
\widehat{\boldsymbol{\theta}}_{L S i}=\left[\sum_{k=k_{\boldsymbol{s}}+1}^{k_{s}+N} \mathrm{z}_{i}\left(k, \tilde{\tau}_{1}, \cdots, \tilde{\tau}_{r}\right) \mathbf{z}_{i}^{T}\left(k, \tilde{\tau}_{1}, \cdots, \tilde{\tau}_{r}\right)\right]^{-1} \cdot\left[\sum_{k=k_{s}+1}^{k_{s}+N} \mathbf{z}_{i}\left(k, \tilde{\tau}_{1}, \cdots, \tilde{\tau}_{r}\right) \xi_{0 \bar{y}_{i}}(k)\right] \quad(i=1,2, \cdots, q)
$$

where Eq.(11) is obtained in the sense of minimizing the following mean squares of the equation error:

$$
V_{L S}\left(\boldsymbol{\theta}_{L S i}, \tilde{\tau}_{1}, \cdots, \tilde{\tau}_{r}\right)=\frac{1}{N} \sum_{k=k_{s}+1}^{k_{s}+N}\left(\xi_{0 \bar{y}_{i}}(k)-\mathrm{z}_{i}^{T}\left(k, \tilde{\tau}_{1}, \cdots, \tilde{\tau}_{r}\right) \boldsymbol{\theta}_{L S i}\right)^{2} \quad(i=1,2, \cdots, q)
$$

When the noise effects can not be neglected, it is well known that, in general, the LS estimates are biased $^{18)}$. In this case we can apply the IV method in a bootstrap manner with the filtered input-output data of the estimated system model as instrumental variables in order to get consistent estimates. The estimated real outputs $\widehat{\bar{x}}_{i}(k)$ can be obtained by the bilinear transformation:

$$
\widehat{\bar{x}}_{i}(k)=\frac{\sum_{h=1}^{r} \sum_{j=1}^{m} \hat{b}_{i h j}\left(\frac{T}{2}\right)^{n-m+j}\left(1+z^{-1}\right)^{n-m+j}\left(1-z^{-1}\right)^{m-j}}{\sum_{j=0}^{n} \hat{a}_{i j}\left(\frac{T}{2}\right)^{j}\left(1+z^{-1}\right)^{j}\left(1-z^{-1}\right)^{n-j}} \bar{u}_{h}\left(k-l_{h}\right) \quad(i=1,2, \cdots, q)
$$

where $\hat{a}_{i j}, \hat{b}_{i h j}$ are the estimates of the system parameters. $l_{h}$ is given by Eq.(8) and if $\Delta_{h} \neq 0$, we have $\widehat{\bar{x}}_{i}(k)$ by the above mentioned interpolation.

Define the IV vectors as follows:

$$
\begin{array}{r}
\mathbf{m}_{i}^{T}\left(k, \tilde{\tau}_{1}, \cdots, \tilde{\tau}_{r}\right)=\left[-\mathbf{m}_{i \widehat{\bar{x}}}^{T}(k), \mathbf{z}_{i \bar{u}_{1}}^{T}\left(k-\tilde{\tau}_{1}\right), \cdots, \mathbf{z}_{i \bar{u}_{r}}^{T}\left(k-\tilde{\tau}_{r}\right)\right] \\
\mathbf{m}_{i \widehat{\bar{x}}}^{T}(k)=\left[\xi_{1 \widehat{\bar{x}}_{i}}(k), \cdots, \xi_{r \widehat{x}_{i}}(k)\right] \quad(i=1,2, \cdots, q)
\end{array}
$$

Using these IV vectors, we can estimate the system parameters by the IV method:

$$
\hat{\boldsymbol{\theta}}_{I V i}=\left[\sum_{k=k_{s}+1}^{k_{s}+N} \mathrm{~m}_{i}\left(k, \tilde{\tau}_{1}, \cdots, \tilde{\tau}_{r}\right) \mathbf{z}_{i}^{T}\left(k, \tilde{\tau}_{1}, \cdots, \tilde{\tau}_{r}\right)\right]^{-1} \cdot\left[\sum_{k=k_{s}+1}^{k_{s}+N} \mathrm{~m}_{i}\left(k, \tilde{\tau}_{1}, \cdots, \tilde{\tau}_{r}\right) \xi_{0 \bar{y}_{i}}(k)\right] \quad(i=1,2, \cdots, q)
$$

We should notice that the IV estimates do not minimize the mean squares of the equation error of Eq. $(12)^{19)}$. However if the IV estimates are consistent, they minimize the following mean squares of the output error:

$$
\begin{aligned}
V_{I V}\left(\boldsymbol{\theta}_{I V i}, \tilde{\tau}_{1}, \cdots, \tilde{\tau}_{r}\right) & =\frac{1}{N} \sum_{k=k_{s}+1}^{k_{s}+N}\left(\bar{y}_{i}(k)-\overline{\bar{x}}_{i}(k)\right)^{2} \\
& =\mathrm{E}\left[\left(\bar{y}_{i}(k)-\widehat{\bar{x}}_{i}(k)\right)^{2}\right] \\
& =\mathrm{E}\left[\left(\bar{x}_{i}(k)-\widehat{\bar{x}}_{i}(k)\right)^{2}\right]+\mathrm{E}\left[\bar{v}_{i}^{2}(k)\right] \\
& =\mathrm{E}\left[\bar{v}_{i}^{2}(k)\right] \quad(i=1,2, \cdots, q)
\end{aligned}
$$

where $\mathrm{E}[\cdot]$ denotes the expectation. Therefore in the next section we calculate the fitness value from the mean squares of the output error of Eq.(16). 


\section{Identification algorithm using the GA}

As mentioned above, in conventional methods the estimates may converge to local optima, or are often affected by measurement noises. In this section, to overcome these problems we propose an identification method using the GA, by which the estimates of the time-delays and the system parameters can arrive at the global optimal point without being locked at local optima. Both the time-delays and the system parameters are identified here.

At first we generate an initial population which consists of binary bit strings as candidates of the time-delays. Then the system parameters are estimated by the IV method for each of candidates of the time-delays which are decoded from the strings. And then the fitness values mapped from the mean squares of the output errors are calculated. Furthermore, the genetic operations, i.e. reproduction based on fitness values, crossover and mutation are repeated so that the average fitness value of the candidate population of the time-delays increases, in order to obtain the optimal estimates of the time-delays and the system parameters. The concrete algorithm is described as follows:

(1) Coding of time-delays $\tau_{h}(h=1,2, \cdots, r)$ :

Code the time-delays $\tau_{h}$ into binary bit strings of $r L$ bits as follows:

$$
\overbrace{\underbrace{1101 \cdots 1010}_{\tau_{1}} \underbrace{0110 \cdots 0101}_{\tau_{2}} \cdots \underbrace{\text { rLbits }}_{\tau_{r}} \cdots 01 \cdots 1100}^{1001 \cdots}
$$

(2)Initial population:

Generate an initial population of $M$ strings randomly.

\section{(3) System parameter identification:}

Identify the system parameters from Eq.(15) by the IV method for each of candidates of the time-delays which are decoded from the strings. The IV vectors of Eq.(14) are given by using the estimated time-delays $\hat{\tilde{\tau}}_{h}^{*}$ and the estimated system parameters $\hat{a}_{i j}^{*}, \hat{b}_{i h j}^{*}$, which have the best fitness value over all the past generations.

In the early generations it is often impossible to calculate Eq.(16), since some output errors of individual strings diverge because some estimated models are unstable. In this case we treat these individual strings as dead genes. However, this treatment may cause early convergence due to loss of the diversity in a population. Therefore we use the LS method until prespecified $G_{L S}$-th generation, then the IV method is started at $\left(G_{L S}+1\right)$-th generation.

\section{(4) Fitness value calculation:}

The mean squares error is mapped into the fitness value for each of the strings. That is, maximizing the fitness value corresponds to minimizing the mean squares error.

Using the mean squares of the output error, the fitness value function is given by

$$
J_{I V}\left(\widehat{\boldsymbol{\theta}}_{I V 1}, \cdots, \widehat{\boldsymbol{\theta}}_{I V q}, \widehat{\tilde{\tau}}_{1}, \cdots, \widehat{\tilde{\tau}}_{r}\right)=\frac{1}{\beta+\frac{1}{N} \sum_{i=1}^{q} w_{i} \sum_{k=k_{s}+1}^{k_{s}+N}\left(\bar{y}_{i}(k)-\overline{\bar{x}}_{i}(k)\right)^{2}}
$$


where $\widehat{\bar{x}}_{i}(k)(i=1,2, \cdots, q)$ are the estimated real outputs and $\beta$ is the constant which adjusts the shape of the curve of the fitness value function ( $\beta$ is chosen to be 0.01 in the example). $\dot{w}_{i}(i=1,2, \cdots, q)$ are weighting factors, which are usually designed to be 1 . In the case when the powers of the outputs are quite different from each other, it is recommendable to adjust $w_{i}$ so that each output contributes to the fitness function equally.

If the estimated model is unstable, then $J_{I V}$ should be zero.

\section{(5) Reproduction}

In this paper reproduction is implimented as a linear search through roulette wheel slots weighted in proportion to the fitness value of the individual string in the old generation. Each one of the old population is reproduced with the probability of $J_{I V}\left(\widehat{\boldsymbol{\theta}}_{I V 1}, \cdots, \hat{\boldsymbol{\theta}}_{I V q}, \widehat{\tilde{\tau}}_{1}, \cdots, \widehat{\tilde{\tau}}_{r}\right) / \bar{J}_{I V}$. Here $\bar{J}_{I V}$ is the sum of the fitness value.

Moreover, we use a method called elitist reproduction which guarantees that the best string is likely to survive from generation to generation.

\section{(6) Crossover}

Pick up two strings randomly and decide whether or not to cross them over according to the crossover probability $P_{c}$. If a crossover is required, exchange strings at a crossing position. The crossing position is chosen randomly.

\section{(7) Mutation}

Alter a bit of string ( 0 or 1 ) according to the mutation probability $P_{m}$.

\section{(8) Repetition}

$(3) \sim(7)$ step are repeated from generation to generation so that the average fitness value of the population increases. The best time-delays are identified as the individual string with the best fitness value over all the past generations, and estimates of the system parameters are given by the IV method with the best time-delays.

\section{Illustrative example}

Consider the following two-input two-output time-delay system described by

$$
\begin{aligned}
& {\left[\begin{array}{l}
x_{1}(t) \\
x_{2}(t)
\end{array}\right]=\left[\begin{array}{cc}
\frac{b_{111} p+b_{112}}{p^{2}+a_{11} p+a_{12}} & \frac{b_{121} p+b_{122}}{p^{2}+a_{11} p+a_{12}} \\
\frac{b_{211} p+b_{212}}{p^{2}+a_{21} p+a_{22}} & \frac{b_{221} p+b_{222}}{p^{2}+a_{21} p+a_{22}}
\end{array}\right]\left[\begin{array}{l}
u_{1}\left(t-\tau_{1}\right) \\
u_{2}\left(t-\tau_{2}\right)
\end{array}\right]} \\
& {\left[\begin{array}{llllll}
a_{11} & a_{12} & b_{111} & b_{112} & b_{121} & b_{122}
\end{array}\right]=\left[\begin{array}{llllll}
3.0 & 4.0 & 1.0 & 2.0 & 2.0 & 3.0
\end{array}\right]} \\
& {\left[\begin{array}{llllll}
a_{21} & a_{22} & b_{211} & b_{212} & b_{221} & b_{222}
\end{array}\right]=\left[\begin{array}{llllll}
2.0 & 2.0 & 2.0 & 1.0 & 1.0 & 4.0
\end{array}\right]} \\
& \tau_{1}=2.570 \quad \tau_{2}=9.130
\end{aligned}
$$

The inputs $u_{h}(t)(h=1,2)$ are outputs of a zero-order hold driven by filtered white signals. The sampling period is taken to be $T=0.05$, and $\alpha_{i}$ in $Q_{i}(p) \quad(i=1,2)$ are chosen to be 0.3 equally. Simulation is 
carried out through 20 realizations of the measurement noises, when NSR(noise to signal ratio) $=20 \%$. The results are shown in Table 1. The table includes the mean and deviation of the estimates. The design parameters of the GA are given as follows:
(1) population size $M=80$
(2) string length $r L=28$
(3) generation number $G=40$
$\left(G_{L S}=5\right)$
(4) crossover probability $P_{c}=0.8$
(5) mutation probability $P_{m}=0.03$

The search ranges of $\tau_{1}, \tau_{2}$ are $\left[0,\left(2^{14}-1\right) / 1000\right]=[0,16.383]$ respectively, and each resolution is 0.001 .

Table 1 shows that the method combining the IV method with the GA yields accurate estimates of the time-delays and the system parameters.

If we attempt to identify this system by searching the time-delays one by one with an accuracy of the resolution of 0.001 , it is not computationally feasible, since $2^{28} \approx 2.7 \times 10^{8}$ times implementations are needed. On the other hand, our method is even feasible on a personal computer, because it requires only $M \times G=3200$ times implementations. Moreover, in the case when we apply the nonlinear estimation method to the system where the time-delays greatly differ from each other like this example, it is very difficult to choose suitable initial values for the parameters. This fact shows that compared with other nonlinear estimation methods, our method is much better.

Table 1: Estimates of the time-delays and system parameters $(N=2000)$

\begin{tabular}{|c|c|c|c|c|c|c|c|}
\hline $\begin{array}{c}\widehat{\tau}_{1} \\
(2.570)\end{array}$ & $\begin{array}{c}\widehat{\tau}_{2} \\
(9.130)\end{array}$ & $\begin{array}{r}\widehat{a}_{11} \\
(3.0)\end{array}$ & $\begin{array}{r}\widehat{a}_{12} \\
(4.0)\end{array}$ & $\begin{array}{r}\hat{b}_{111} \\
(1.0)\end{array}$ & $\begin{array}{r}\hat{b}_{112} \\
(2.0) \\
\end{array}$ & $\begin{array}{r}\hat{b}_{121} \\
(2.0) \\
\end{array}$ & $\begin{array}{r}b_{122} \\
(3.0) \\
\end{array}$ \\
\hline 2.571 & 9.130 & 2.9804 & 3.9003 & 1.0113 & 1.9381 & 2.0077 & 2.9176 \\
\hline \multirow[t]{3}{*}{ \pm 0.005} & \pm 0.005 & \pm 0.0466 & \pm 0.2008 & \pm 0.0279 & \pm 0.1076 & \pm 0.0417 & \pm 0.1675 \\
\hline & & $\begin{array}{r}\widehat{a}_{21} \\
(2.0)\end{array}$ & $\begin{array}{r}\widehat{a}_{22} \\
(2.0)\end{array}$ & $\begin{array}{r}\hat{b}_{211} \\
(2.0)\end{array}$ & $\begin{array}{r}b_{212} \\
(1.0)\end{array}$ & $\begin{array}{r}\hat{b}_{221} \\
(1.0) \\
\end{array}$ & $\begin{array}{r}\widehat{b}_{222} \\
(4.0) \\
\end{array}$ \\
\hline & & $\begin{array}{r}2.0197 \\
\pm 0.0393\end{array}$ & $\begin{array}{r}2.0123 \\
\pm 0.0359\end{array}$ & $\begin{array}{r}2.0117 \\
\pm 0.0302 \\
\end{array}$ & $\begin{array}{r}1.0092 \\
\pm 0.0456\end{array}$ & $\begin{array}{r}0.9871 \\
\pm 0.0381\end{array}$ & $\begin{array}{r}4.0347 \\
\pm 0.0863\end{array}$ \\
\hline
\end{tabular}

\section{Conclusions}

In this paper we have proposed the identification algorithm combining the IV method with the GA for multi-input, multi-output continuous time-delay systems which, in general, has been considered to be difficult. In our approximated discrete-time estimation model, the time-delays need not be an integral multiple of the sampling period. Due to the advantage of the GA, the estimates of the time-delays and the system parameters can reach the global optimal point without being locked at local optima. Simulation results show that the proposed method yields consistent estimates even in the presence of high measurement noises.

\section{References}

[1] W. X. Zheng and C. B. Feng: Identification of Stochastic Time Lag Systems in the Presence of Colored Noise; Automatica, Vol.26, No.4, pp.769 779 (1990)

[2] W. X. Zheng and C. B. Feng: Optimizing Search-Based Identification of Stochastic Time-Delay Systems; International Journal of Systems Science, Vol.22, No.5, pp.783 792 (1991) 
[3] G. Ferretti, C. Maffezzoni and R. Scattolini: Recursive Estimation of Time Delay in Sampled Systems; Automatica, Vol.27, No.4, pp.653 661 (1991)

[4] S. Sagara, Z. J. Yang, K. Wada: Identification of Continuous-Time Systems (in Japanese); Systems, Control and Information, Vol.37, No.5, pp.284 290 (1993)

[5] P. J. Gawthrop, and M.'T. Nihtilä: Identification of Time-Delays using a polynomial identification method; System and Control Letters, Vol.5, No.4, pp.267 271 (1985)

[6] E. W. Bai and D. H. Chyung: Improving Delay Estimates Derived from Least-Square Algorithms and Padé Approximations; International Journal of Systems Science, Vol.24, No.4, pp.745 756 (1993)

[7] M. Agarwal and C. Canudas: On-line Estimation of Time Delay and Continuous-Time Process Parameters; International Journal of Control, Vol.46, No.1, pp.295 311 (1987)

[8] Z.Y. Zhao and S. Sagara: Consistent Estimation of the Time Delay in Continuous-Time Systems; Transactions of the society of instrument and control engineers, Vol.27, No.1, pp.64 69 (1991)

[9] D. C. Saha and G. P. Rao: Identification of Lumped Linear Systems in the Presence of Small Unknown Delays - the Poisson Moment Functional Approach; International Journal of Control, Vol.33, No.5, pp.945 951 (1981)

[10] G. P. Rao and L. Sivakumar: Identification of Time-Lag Systems via Walsh Functions; IEEE Trans.on Automatic Control, Vol.AC-24, No.5, pp.806 808 (1979)

[11] P. J. Gawthrop, M. T. Nihtilä and A. Besharati-Rad: Recursive Parameter Estimation of Continuous-Time Systems with Unknown Time Delay; Control-Theory and Advanced Technology, Vol.5, No.3, pp.227 248 (1989)

[12] A. E. Pearson and C. Y. Wuu: Decoupled Delay Estimation in the Identification of Differential Delay Systems; Automatica, Vol.20, No.6, pp.761 772 (1984)

[13] D. E. Goldberg: Genetic Algorithms in Search, Optimization, and Machine Learning; Addison-Wesley Publishing Company, Inc. (1989)

[14] J. E. Lansberry, L.Wozniak and D. E. Goldberg: Optimal Hydrogenerator Governor Tuning with a Genetic Algorithm; IEEE Trans. on Energy Conversion, Vol.7, No.4, pp.623 630 (1992)

[15] M. Tanaka, T. Hattori and T. Tanino: Jumped Detection and Identification of Linear Systems by the Genetic algorithm (in Japanese); Transactions of the society of instrument and control engineers, Vol.28, No.11, pp.1383 1385 (1992)

[16] S. Sagara, Z. J. Yang, K. Wada and T. Tsuji: Parameter Identification and Adaptive Control of Continuous Systems with Zero-Order Hold; IFAC World Congress, Sydney (1993)

[17] S. Sagara, K. Wada and K. Nakano: Fundamentals of Digital Control (in Japanese); CORON A PUBLISHING CO., LTD., pp.127 128 (1992)

[18] T. Nakamizo: Signal Analysis and System Identification (in Japanese); CORONA PUBLISHING CO., LTD. (1992)

[19] Söderström, T. and Stoica, P.: Comparison of Some Instrumental Variable Methods Consistency and Accuracy Aspects; Automatica, Vol.17, No.1, pp.101 115 (1981) 